\title{
A Novel Drive Option for Piezoelectric Ultrasonic Transducers
}

\author{
Diana Engelke, ${ }^{1}$ Bernd Oehme, ${ }^{2}$ and Jens Strackeljan ${ }^{1}$ \\ ${ }^{1}$ Institute of Mechanics, Otto-von-Guericke University Magdeburg, Universitaetsplatz 2, 39106 Magdeburg, Germany \\ ${ }^{2}$ Division of Instruments, Sirona Dental Systems GmbH, Fabrikstrasse 31, 64625 Bensheim, Germany
}

Correspondence should be addressed to Diana Engelke, diana.engelke@ovgu.de and Jens Strackeljan, jens.strackeljan@ovgu.de

Received 4 March 2011; Accepted 17 May 2011

Academic Editor: Jing-Song Hong

Copyright (C) 2011 Diana Engelke et al. This is an open access article distributed under the Creative Commons Attribution License, which permits unrestricted use, distribution, and reproduction in any medium, provided the original work is properly cited.

\begin{abstract}
This paper concentrates on ultrasonic transducers, which are driven by piezoelectric ceramic rings that are arranged in a stack. A novel drive option, where the stack contains a new type of divided piezoelectric rings, is analyzed using the finite element method, prototyped, and tested. To gain a better sense of the vibration behavior, the studies focus initially on one ring and subsequently on the different possibilities to assemble the transducer. The investigations point out that natural bending frequencies can be excited at the transducer. Thus, multiple vibration directions of the tip can be controlled, what can be advantageous for instance in dental applications.
\end{abstract}

\section{Introduction}

Piezoelectric actuators are applied in various engineering applications, for example, in the fields of nanopositioning, ultrasonics (ultrasonic welding), ultrasonic motors and diesel injection. These transducers use the piezoelectric effect for the conversion of electrical energy to mechanical displacement. They have the advantage to combine high speed and high positioning precision. An application example is the sandwich or Langevin transducer. The transducer or parts of it have been analyzed analytically in [1-3] and by means of the Finite Element Method (FEM) in $[4,5]$ as well as by experimental studies in $[6,7]$. This paper investigates a dental ultrasonic scaler by means of the FEM and validates the data via experiments.

A dental ultrasonic scaler (Figure 1) is a hand-held instrument for the mechanical removal of dental plaque and calculus from the teeth. It is driven by piezoelectric ceramic rings that are arranged in a stack and that induce axial vibrations. These vibrations are transmitted through the sonotrode (or horn), which amplifies them, to the tip. The tip is shaped in a way that it increases the amplitudes further, so that the resulting oscillation can remove the dental plaque. The resonator absorbs the reaction forces that emerge during operation and the o-rings serve as bearing. The dentist uses the scaler during the periodontal therapy or the professional dental cleaning. A laboratory study [8] revealed that the tooth surface takes little damage if the scaler tip oscillates in parallel to it (Figure 2 left).

Today, there are two types of scaler probes on the market, the straight and the bent probes. The straight probes are intended to vibrate with a linear movement in the longitudinal direction (the $\mathrm{x}$ direction or $0^{\circ}$ in Figure 1 ) of the scaler. Recent studies $[6,9]$ showed that this vibration behavior is not always ensured and the tips may oscillate elliptically and with a lateral component. However, this is generally unintended [10]. By contrast, the bent probes are designed to oscillate asymmetrically to the longitudinal plane, since the geometrically shape is asymmetrical [9]. For both kinds of probes an elliptical vibration pattern of the tip is possible, depending on the shape and design of the probe. In this paper, the main vibration direction of the tip will be evaluated, because previous measurements of the considered probe revealed that the elliptical component is negligible.

However, all of the above mentioned scalers oscillate in solely one main direction. It is likely that the dentist cannot permanently ensure a parallel alignment between the tip and the tooth (Figure 2 middle), because of the limited movement abilities in the patient's oral cavity. This harms the dental substance unnecessarily. So it is desirable that the tip is able to oscillate in multiple spatial directions, as indicated in Figure 1. Moreover, the vibration directions shall 


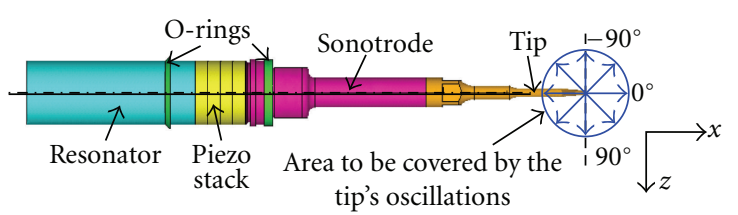

Figure 1: Dental ultrasonic scaler (upper view).
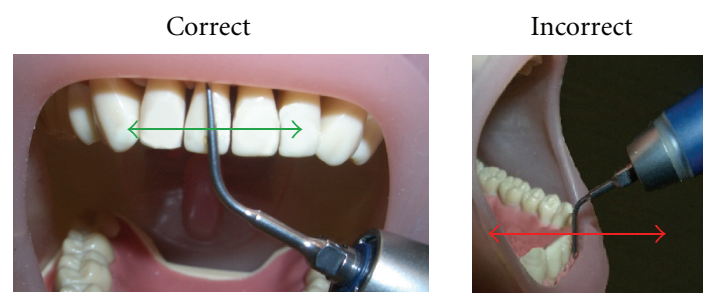

$\longleftrightarrow$ Correct (tangential) oscillation

$\longleftrightarrow$ Incorrect handling (perpendicular to the tooth)

Figure 2: Handling of the scaler.

be controlled individually, so that it is possible to predict the movement of the tip precisely. Hence, with an appropriate control the tip is able to work permanently in parallel to the tooth surface. A device that can provide such a control is presented in [11]. The software of this system includes a detection mode, which discriminates dental calculus deposits and clean tooth surfaces $[12,13]$. This algorithm can be upgraded to detect the angle between the oscillating tip and the tooth surface and to control the drive frequency of the scaler, so that the tip oscillates parallel to the tooth surface.

One possibility to induce multidimensional vibration in multiple spatial directions to the tip is the use of piezoceramic rings that consist of two segments which are oppositely polarized. The idea to integrate divided rings into an ultrasonic transducer comes up first in [14]. They induce longitudinal-mode and flexural-mode vibrations in a one-dimensional construction of a transducer system by means of two sets of piezoceramics. One set consists of longitudinally polarized circular piezoceramic rings that are used to generate the longitudinal oscillations. The other one comprises half rings, which are polarized in opposite directions and thus generate the flexural-mode vibrations. The physical dimensions of the transducer are varied so that both vibration modes are at the same resonant frequency. The resulting vibrations are in the range of some micrometers. An analytical solution for this problem is presented in [15] using the example of a slender rod.

In the context of ultrasonic scalers, the idea to integrate divided piezoelectric ceramic rings comes up first in [16]. The generated vibrations result in a transverse movement of the tip. The scaler transforms a longitudinal excitation to a perpendicular oscillation of the tip, but it is still only one vibration direction. The idea to excite multidimensional vibrations of a scaler tip by using divided piezoceramic rings is first risen in [17].

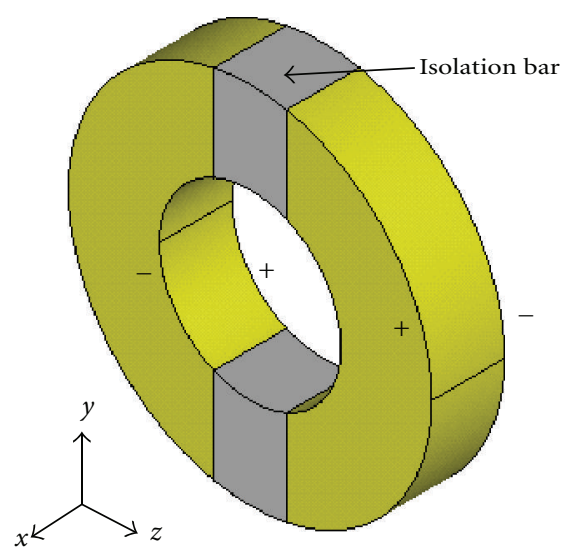

FIGURE 3: Model of a new piezoceramic ring.

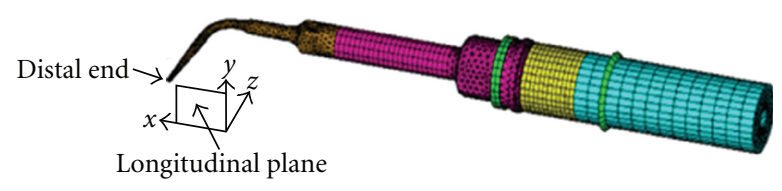

Figure 4: ANSYS model of the ultrasonic scaler.

\section{Materials and Methods}

The starting point for the investigations is a conventional scaler with a straight tip. In this scaler new, divided piezoceramic rings, which have the same dimensions as the original ones, are integrated. These rings are diametrically separated by an isolation bar and the halves are oppositely polarized. A model of one ring is shown in Figure 3. The directions of polarization are indicated.

2.1. Finite Element Analysis. The behavior of a piezoceramic ring and the whole system of the ultrasonic scaler is studied via a 3D FE model using ANSYS. The simulation model of the ultrasonic scaler is presented in Figure 4. The material data is taken according to DIN specifications and adjusted to the model. The potential difference at each piezoceramic is $130 \mathrm{~V}$. The model does not take into account the prestress of the piezostack during the assembly or the temperature raise of the ceramics during operation, which could lead to a variation of vibration amplitudes and a frequency shift. However, it will become apparent that the model predicts well the behavior of the scaler.

Furthermore the deformation of one piezoceramic will be simulated. The manufacturer PI Ceramic $\mathrm{GmbH}$ provides the material data for the piezoceramic. A voltage differential of $100 \mathrm{~V}$ is applied.

An analysis is performed regarding the deformation of the new piezoceramic as well as the vibration behavior of the scaler, meaning the amplitude at the distal end in longitudinal and transverse direction. Thereby a scaler with two original and two divided piezoceramic rings in the stack is examined, and the influence of the position and rotation of the divided piezoceramics is pointed out. 


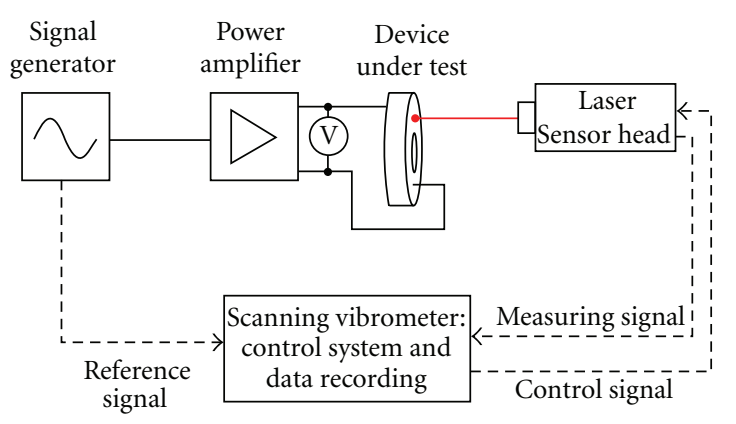

FIGURE 5: Displacement measurement of one piezoelectric ring.

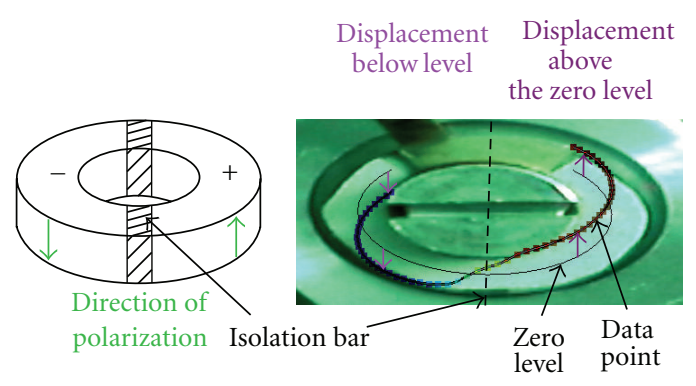

FIGURE 6: Displacement of one new piezoceramic ring.

2.2. Displacement Measurement. The PSV-400 Scanning Laser Vibrometer (Polytec $\mathrm{GmbH}$ ) is employed to characterize the deformation of the original and the divided piezoceramics. This device measures the vibration velocity at multiple points on the surface of a ceramic. Figure 5 shows the test setup. The piezoceramic is excited with $100 \mathrm{~V}$ AC voltage at $10 \mathrm{kHz}$ frequency. The measurement of the vibration velocity takes place in the time domain at ca. 40 points distributed over the periphery of the ring. The points are predefined via the laser's data management system. This system takes the control during the measurement.

For quantifying the amplitude of the tip's distal end, different measuring systems could be used. Measurements can be carried out for instance with a Scanning Laser Vibrometer [7], which contactlessly measures the motion of the tip. In this work, the measurements are performed with an electrooptical displacement transducer, which is a noncontact measuring system as well. For the frequencies that lie within the working range of the scaler, the longitudinal and transversal vibration directions are recorded.

\section{Results and Discussion}

3.1. Deformation of the Piezoceramic Ring. Figure 6 shows the measured vibration velocities of the new type of piezoceramics. Each colored point represents one measured data point. The displacement of the ceramic is obtained by integrating these values. It is well to see that the halves extend in phase opposition. The half left to the isolation bar contracts while the other one extends with respect to the zero level of the ceramic. This is entirely logical, since the oppositely polarized halves are controlled by the same signal.

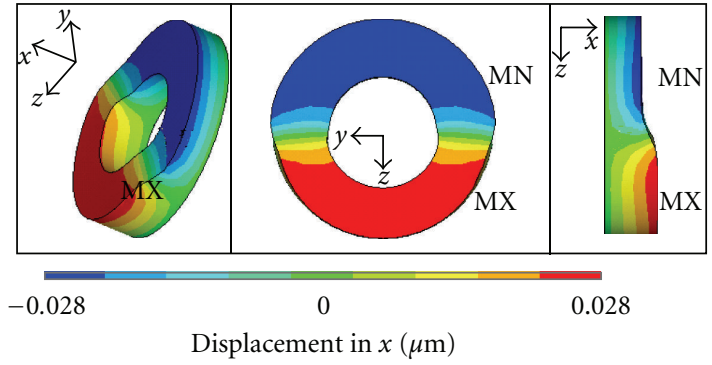

FIGURE 7: FEM calculation of the divided piezoceramic ring.

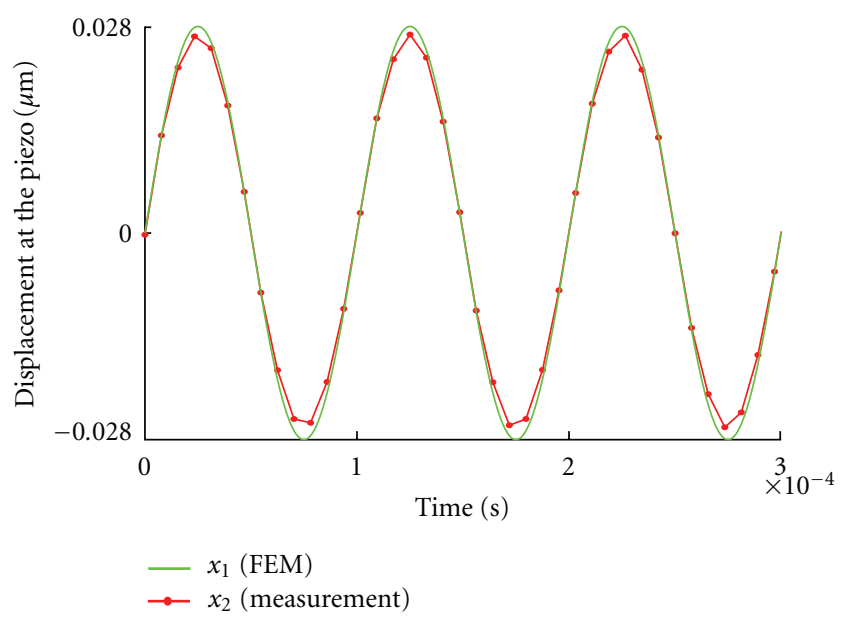

FIGURE 8: Comparison of simulated and experimental displacement data of the divided piezoceramic ring.

The absolute values of the vibration velocity are the same but of opposite signs. The displacement of the conventional piezoceramic is uniform in all measuring points, what means that the upper surface moves evenly. This deformation is obvious and not plotted here.

Figure 7 shows the deformation of a divided piezoceramic that is calculated numerically in ANSYS. The displacement in the polarization direction is marked in color. One sees well that the halves of the ceramic expand in opposite directions.

Figure 8 compares the simulated and measured displacements on the divided piezoceramic. The measuring point is located as far apart as possible from the isolation bar, so that the deflection is maximal. It is well to see that the measurement and simulation data match very well. The maximum deviation of the displacements is less than $7 \%$.

With the understanding of the vibration behavior of a single divided piezoceramic ring, the whole scaler assembly is studied next. Since the simulation and experimental data of the single ring match well, the model of the new piezoceramic is integrated in the model of the overall system. A good compliance of the simulated and measured values is expected.

3.2. Vibration Behavior of the Ultrasonic Scaler. The analysis of the scaler's natural frequencies in the working range re turns two longitudinal mode shapes and two bending 

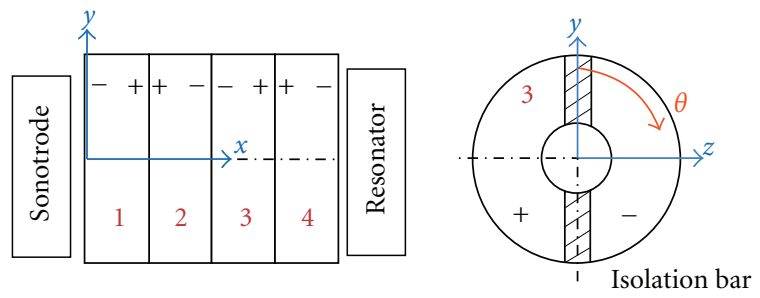

FIgURE 9: Scheme of the piezostack.

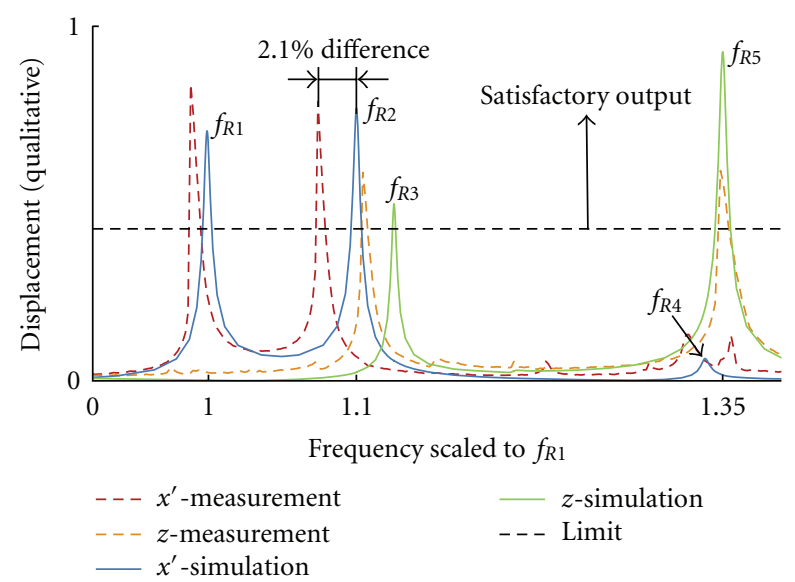

Figure 10: Experimental and simulated displacement of one new scaler.

frequencies. The investigated conventional scaler with its sym-metric structure in regard to the longitudinal plane oscillates solely in longitudinal direction (disregarding possible elliptical movements), whereas the scaler with the divided and conventional piezoceramics excites the system in addition asymmetrically in regard to the longitudinal plane and thus the 2 longitudinal and the 2 bending frequencies can be induced, so that the tip oscillates in multiple directions, depending on the driving frequency.

Figure 9 shows a schematic representation of the piezostack. Each piezoceramic is either original or divided. The divided piezoceramic rings are characterized by the position as well as the rotation angle $\theta$. These two parameters will be varied later, when different configurations of the piezostack are studied.

Figure 10 shows the measured and simulated amplitudes of the distal end for one new scaler (2 divided piezoceramics at positions 2 and $3, \theta=0^{\circ}$ for both). In that diagram $x^{\prime}$ designates the longitudinal and $\mathrm{z}$ the transversal movement of the tip. The qualitative amplitudes are plotted. The tip of the investigated scaler oscillates during normal operation with amplitudes of $90 \mu \mathrm{m}$ or more. Internal tests showed that the output of the scaler is still satisfactory, even if the amplitude amounts to only $30 \%$ of the initial value. This knowledge is used to determine the limit (black dotted line). All amplitudes above this value are considered during the evaluation of an amplitude response. The amplitudes below the limit are not of interest, since they induce a low output.

The difference between the simulated and measured data regarding the eigenfrequencies amounts to maximum

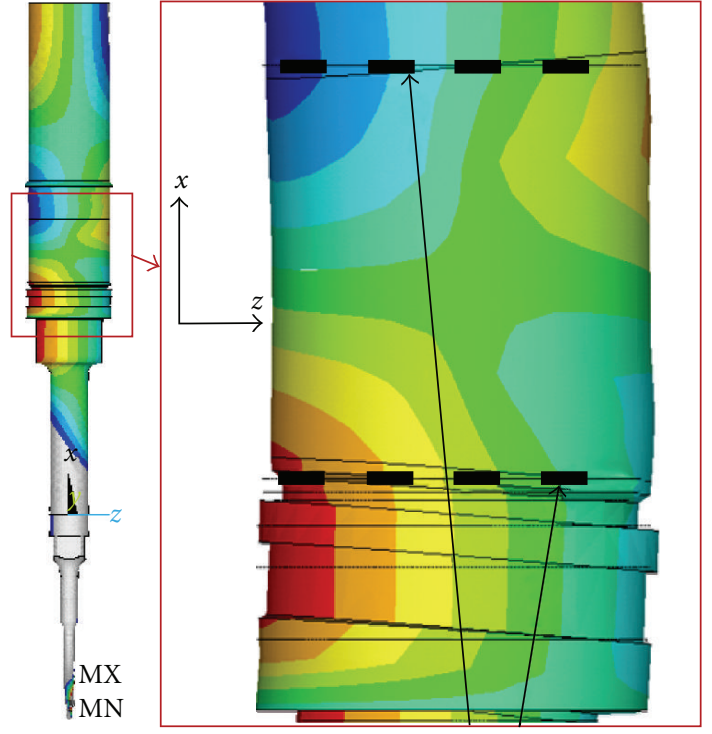

Edges of the undeformed model

FIGURE 11: Deformation of the piezostack.

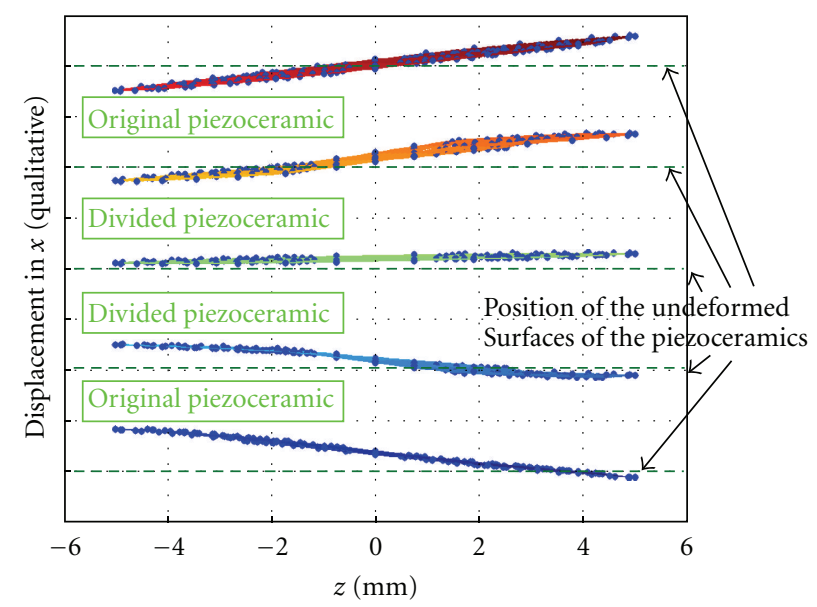

FIGURE 12: Surfaces of the deformed piezoceramics.

$2.1 \%$ (Figure 10). Internal tests showed that the variations of the measured eigenfrequencies of scalers stemming from one series are of the same order of magnitude. Hence the simulation model represents very well the real scaler.

The scaler is simulated at a fixed off-resonance frequency. At this frequency, the calculated deformation of the piezostack depends mainly on the excitation and is not influenced by the resonance effects of the other components. Figure 11 shows the translation of the scaler in longitudinal direction. The deformed piezostack is shown enlarged. Figure 12 illustrates the translation of the outer surfaces of the four piezoceramics in the stack. The plot is derived from the simulation results in ANSYS. The values for the nodal displacement on the outer surface of each piezoceramic are read out and plotted enlarged.

It can be seen clearly that the two divided piezoceramic rings deform asymmetrically in regard to the longitudinal plane $(z=0)$. This is the same behavior that the single 


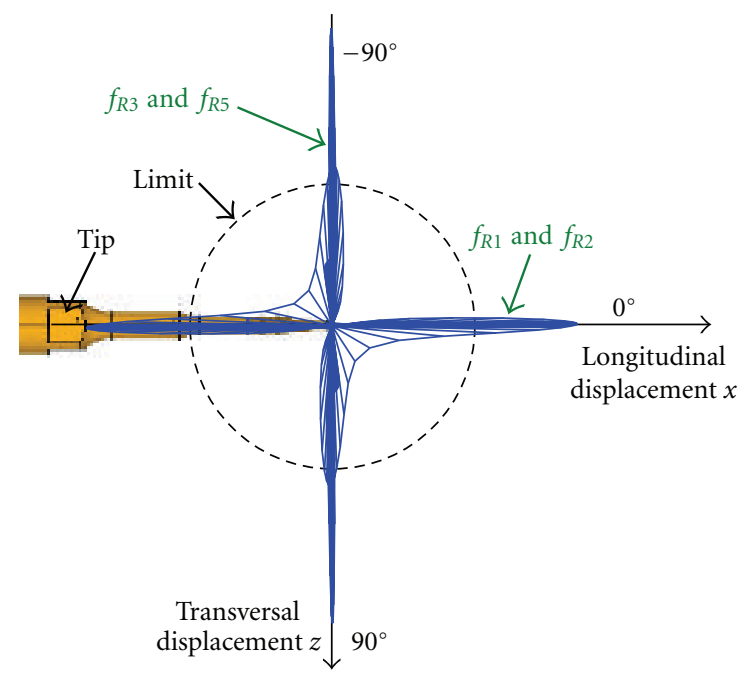

FIGURE 13: Plotted oscillation of one new scaler.

piezoceramic ring has displayed (Figure 7). The original piezoceramic rings extend, as expected, in only one direction. The above comment implies that the use of divided ceramics leads to an asymmetric excitation, which induces flexural vibrations at the associated eigenfrequencies. The original piezoceramics induce longitudinal vibrations. As a consequence, the tip performs longitudinal and transversal movements, depending on the excitation frequency of the scaler.

The results show that the simulated and measured data match very well for a single divided piezoceramic ring as well as for the new scaler assembly. Hence, further studies can be performed on the simulation model without the need for immediate experimental validation.

Based on the obtained results for the scaler assembly the influence of the position and the rotation angle $\theta$ of the divided piezoceramic rings (Figure 9) are studied. Calculations are performed on scalers, the stacks of which contain 2 divided piezoceramics at various positions and rotation angles. The results of these calculations provide the information that it is worthwhile to study the rotation angles $0^{\circ}$ and $180^{\circ}$. For these configurations the application tip oscillates with maximum amplitudes in the longitudinal and transversal direction. An exemplary plot of the oscillations that the scaler from Figure 10 performs is shown in Figure 13. The diagram shows the vibration amplitudes and the corresponding angles. It is well to see that the tip oscillates in more than one direction depending on the driving frequency. This is an improvement compared with the scalers, which are on the market today. The new scaler is able to oscillate in two directions perpendicular to each other. Hence, if the worst were to happen, that is, the longitudinally oscillating tip is positioned perpendicular to the tooth (see Figure 2), the new scaler will be driven at frequency $f_{R 3}$, so that the tip oscillates in parallel to the tooth surface.

However, since the tip should oscillate in as many angles as possible, these two directions are not satisfactory. A solution might be to control the piezoceramics independently, so that the resulting vibrations can be superposed. For that purpose, a redesign of the structure will be necessary.

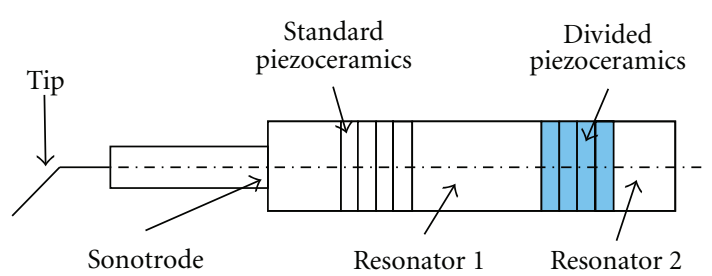

FIGURE 14: Layout of the redesigned structure with two piezostacks.

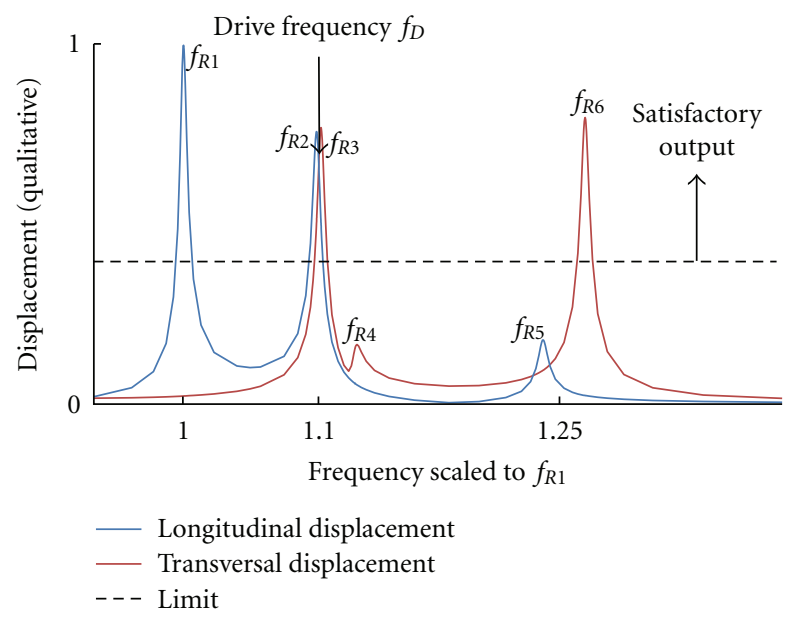

FIGURE 15: Amplitude response of the redesigned structure.

A possible redesign (Figure 14) is simulated in ANSYS. The two piezostacks can be controlled independently, that is, with different frequencies and voltages. The simulation returns the amplitude response (Figure 15). If the stack with the standard piezoceramics is excited, the considered structure vibrates only in the longitudinal direction. Otherwise, if the stack with the divided piezoceramics is excited, the tip performs transversal oscillations. These two amplitude responses can be superposed, when both stacks are controlled with the same frequency. The amplitudes of each vibration direction depend on the applied voltages. In the example, the frequencies for the longitudinal $\left(f_{R 2}\right)$ and flexural vibrations $\left(f_{\mathrm{R} 3}\right)$ are very close. For driving the structure, the frequency $f_{D}$ is chosen, because it allows to superpose the oscillation directions. The amplitudes are scaled with the factors $\mathrm{a}_{x}, \mathrm{a}_{z} \in[0,1]$. An example of the resulting oscillations of the application tip is plotted in Figure 16. It is well to see that all vibration angles between $0^{\circ}$ and $90^{\circ}$ can be controlled if the excitation frequencies are in phase. If they are out of phase, the oscillations cover the angles between $0^{\circ}$ and $-90^{\circ}$. Consequently, this new generator adjusts the vibration direction of the tip in a way that the tip is able to oscillate always in parallel to the tooth surface, no matter how the scaler is positioned with respect to that surface.

\section{Conclusion}

This paper demonstrates the benefits to simulate an ultrasonic dental scaler by means of the FEM. The simulated and measured frequency responses match very well. On this 


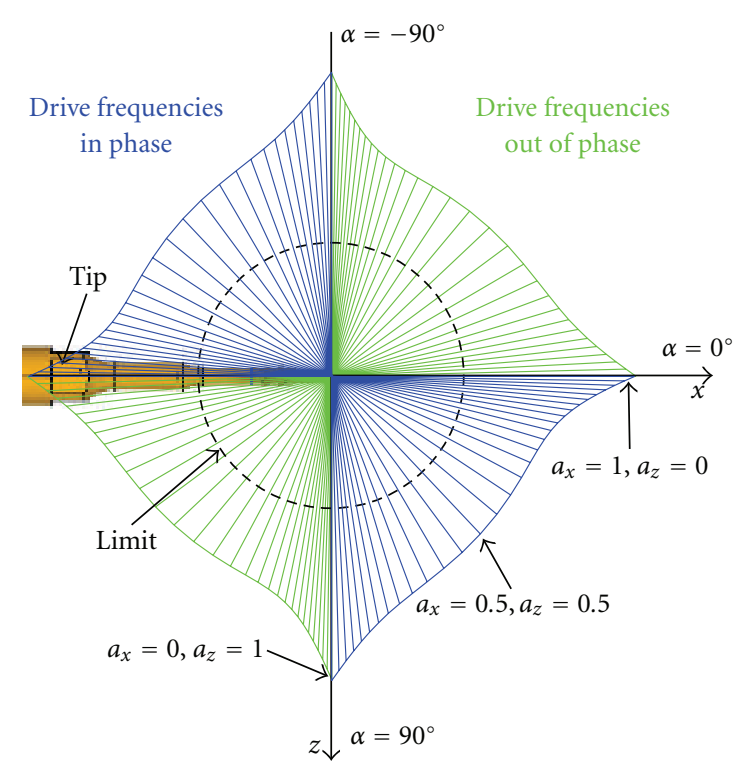

FIgURE 16: Plotted oscillations of the redesigned structure.

basis investigations of new versions are performed. They reveal that the tip is able to oscillate in multiple vibration directions, which can be controlled individually, if the scaler is excited asymmetrically, for example, by means of divided piezoceramics. A redesign of the original transducer shows the potentialities of the divided piezoceramics. By driving the ceramics of the transducer independently, the tip is able to oscillate in all spatial directions. This allows a gentle and painless treatment, because the vibration direction of the tip can be adjusted precisely, depending on the present angle between the tip and the tooth surface.

\section{References}

[1] A. Iula, R. Carotenuto, M. Pappalardo, and N. Lamberti, "An approximated 3-D model of the Langevin transducer and its experimental validation," Journal of the Acoustical Society of America, vol. 111, no. 6, pp. 2675-2680, 2002.

[2] D. D. Mančić and G. Z. Stančić, "New three-dimensional matrix models of the ultrasonic sandwich transducers," Journal of Sandwich Structures and Materials, vol. 12, no. 1, pp. 63-80, 2010.

[3] S. Sherrit, B. P. Dolgin, Y. Bar-Cohen, D. Pal, J. Kroh, and T. Peterson, "Modeling of horns for sonic/ultrasonic applications," in Proceedings of the IEEE Ultrasonics Symposium, pp. 647-651, October 1999.

[4] D. H. Johnson and D. Pal, "Simulation of an ultrasonic piezoelectric transducer," in Proceedings of the 9th International ANSYS Conference and Exhibition, Pittsburgh, Pa, USA, August 2000.

[5] H. Allik, K. M. Webman, and J. T. Hunt, "Vibrational response of sonar transducers using piezoelectric finite elements," Journal of the Acoustical Society of America, vol. 56, no. 6, pp. 1782-1791, 1974.

[6] S. C. Lea, B. Felver, G. Landini, and A. D. Walmsley, "Threedimensional analyses of ultrasonic scaler oscillations," Journal of Clinical Periodontology, vol. 36, no. 1, pp. 44-50, 2009.
[7] S. C. Lea, G. Landini, and A. D. Walmsley, "Vibration characteristics of ultrasonic scalers assessed with scanning laser vibrometry," Journal of Dentistry, vol. 30, no. 4, pp. 147-151, 2002.

[8] T. Flemming, G. Petersilka, and A. Mehl, "The effect of working parameters on root substance removal using a piezoelectric ultrasonic scaler in vitro," Journal of Clinical Periodontology, vol. 69, pp. 547-553, 1998.

[9] A. Janner, Schwingungsverhalten verschiedener Schall- und Ultraschallansätze und Empfehlungen für die klinische Anwendung am Patienten, dissertation, LMU München, 2003.

[10] EMS, "Piezon NO PAIN," Brochure, 2011, http://wwwemscompany.com/media/piezon_master_700/FA-322_EN_ed_2009-03_Brochure\%20Piezon\%20Master\%20700.pdf.

[11] J. Strackeljan et al., "Ein neuartiges dentalgerät mit fuzzyklassifikator," in Proceedings of the Information-Mining und Wissensmanagement in Wissenschaft und Technik, Tagungsband AFN-Symposium, pp. 66-84, 2008.

[12] B. Oehme, Klassifikation von zahnoberflächen mittels ultraschallanregung - ein adaptives verfahren zur mustererkennung, dissertation, TU Clausthal, 2004.

[13] G. Meissner, B. Oehme, J. Strackeljan, and T. Kocher, "A new Clinical subgingival calculus detection with a smart ultrasonic device: a pilot study," Journal of Clinical Periodontology, vol. 35, no. 2, pp. 126-132, 2008.

[14] Y. Watanabe, Y. Tsuda, and E. Mori, "Longitudinal-flexural complex-mode ultrasonic high-power transducer system with one-dimensional construction," Japanese Journal of Applied Physics, Part 1, vol. 32, no. 5, pp. 2430-2434, 1993.

[15] S. Lin, "Study on the Langevin piezoelectric ceramic ultrasonic transducer of longitudinal-flexural composite vibrational mode," Ultrasonics, vol. 44, no. 1, pp. 109-114, 2006.

[16] Duerr Dental GmbH \& Co. KG, “Antriebselement," DE application 102005044074A1, 2007.

[17] Sirona Dental Systems GmbH, "Dentale Ultraschallvorrichtung und Verfahren zum Betrieb einer dentalen Ultraschallvorrichtung," DE application 102007053544A1, 2009. 

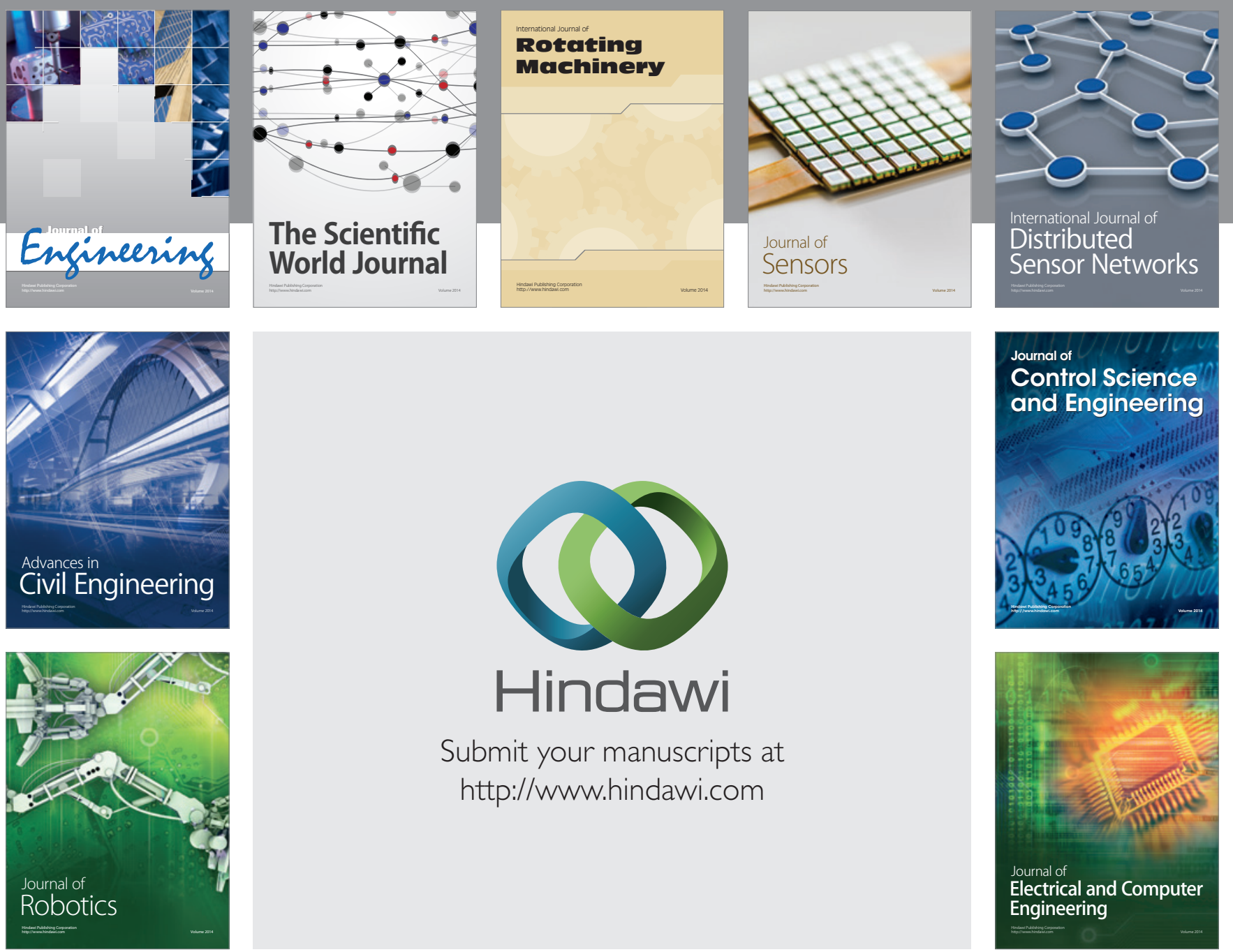

Submit your manuscripts at

http://www.hindawi.com
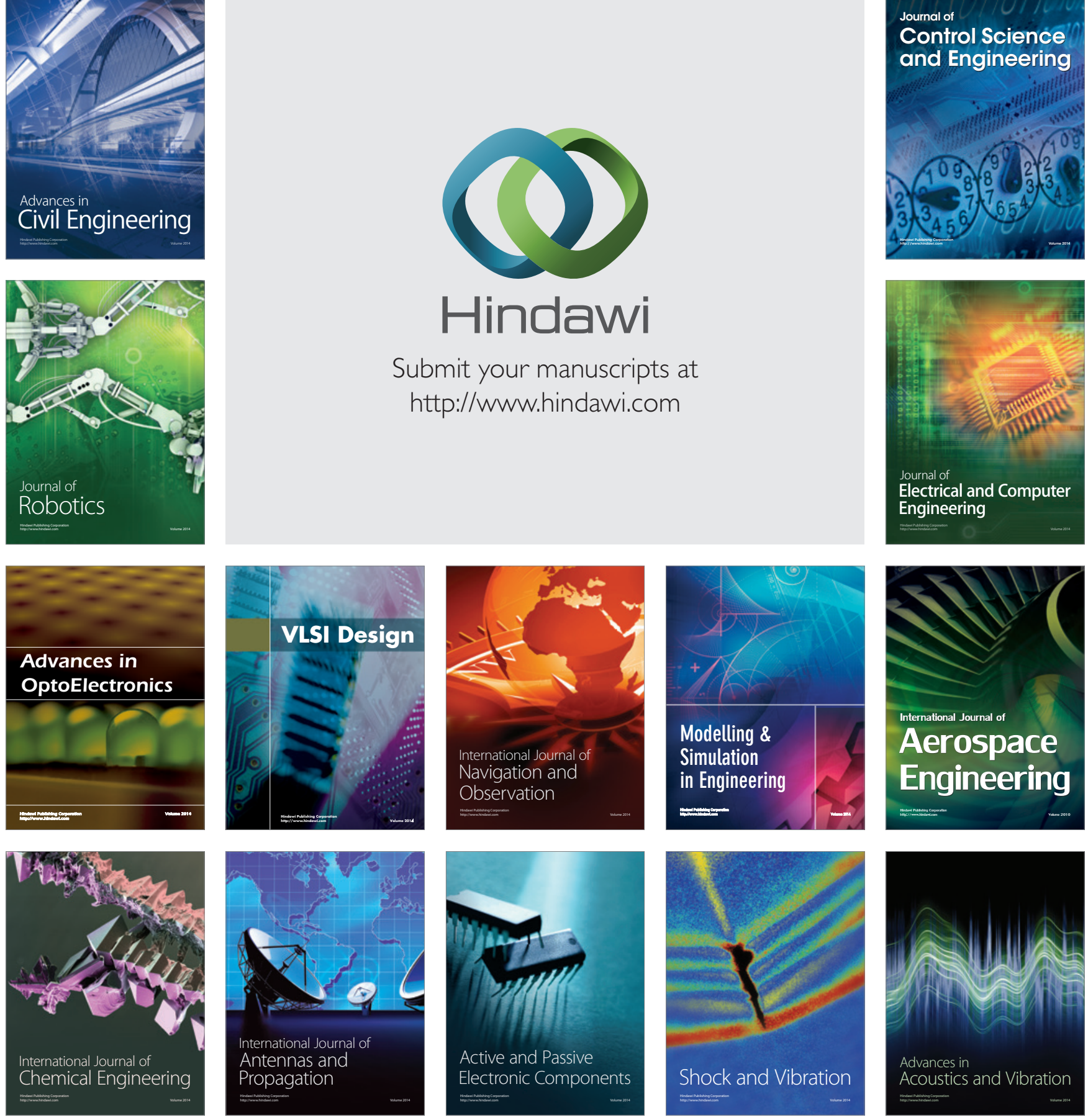